\title{
Modifications des horaires de garde : conséquences sur la perception par les résidents de leur qualité de vie et de la sécurité des patients
}

\author{
Effect of on-call schedule changes: impact on resident perceived quality \\ of life and patient safety
}

\author{
Marie-Hélène LIZOTTE ${ }^{1}$, Marie-Christine LAMY ${ }^{2}$, Benoit CARRIÈRE ${ }^{1}$ \\ 1 Département de pédiatrie, CHU Sainte-Justine, Montréal, Canada \\ 2 Département de pédiatrie, Hôpital Pierre-Legardeur, Montréal, Canada
}

Manuscrit soumis le 12 janvier 2014 ; commentaires éditoriaux formulés aux auteurs le 28 janvier 2014 ; accepté pour publication le 21 février 2014

\begin{abstract}
Mots-clés
Horaires de gardes ; résidents ; sécurité des patients ; qualité de vie ; pédiatrie
\end{abstract}

\section{Keywords} On-call schedule; residents; patient safety; quality or life; pediatrics
Résumé - Contexte : En 2012, les médecins résidents du Québec ont cessé de faire des quarts de travail de plus de 16 heures consécutives. But : Évaluer les conséquences sur la perception de la qualité de vie et de la sécurité des soins. Population : Résidents du programme de résidence en pédiatrie de l'Université de Montréal. Méthodes : Questionnaire administré en ligne avant et après le changement des horaires de garde. Le premier questionnaire $(\mathrm{Q} 1)$ a été réalisé alors que les résidents effectuaient des gardes de 24 heures. Le second questionnaire (Q2) a été réalisé après la limitation du nombre d'heures de travail (maximum : 12 heures consécutives). Résultats : Taux de réponse : 0,85 pour le Q1, 0,80 pour le Q2. Le nombre moyen d'heures de travail par semaine en milieu hospitalier était le même avant et après le changement. Pour $62 \%$ des résidents, le nouveau système allait améliorer leur qualité de vie et $84 \%$ estiment que tel a été le cas. Pour $96 \%$ des répondants, la sécurité des patients s'est améliorée après le changement du système de garde. Conclusion : La suppression des gardes de 24 heures au sein d'un programme de résidence en pédiatrie a des impacts positifs sur la perception de qualité de vie et l'impression de sécurité et de qualité des soins aux patients.

Abstract - Background: In 2012, the province of Quebec called for the elimination of resident work shifts in excess of 16 hours. Objective: To evaluate the impact of an on-call system change in a pediatrics residency program with respect to perceived quality and safety of care provided to patients, as well as quality of life for residents involved. Method: A study using web-based pre- and post- surveys was carried out. The initial survey (IS) was completed with residents involved in 24-hour calls. The second survey (SS) was sent out after the on-call system switched to 12-hour calls. Results: Response rate of 0.85 (IS) and 0.8 (SS). The mean number of hours per week spent in hospital remained unchanged before and after 
the system switch. Quality of life improved for $62 \%$ of initial responders when asked about 12-hour calls; $84 \%$ felt the same way after the on-call system change was implemented. Perceived patient safety improved according to $96 \%$ of responders in SS. Conclusion: The oncall system change had a mostly positive significant impact in the pediatrics residency program with respect to quality of life, perceived patient safety and quality of care.

\section{Introduction}

\section{Contexte et problématique}

L'organisation du travail des résidents (nommés internes dans certains pays francophones) en médecine est remise en cause depuis la fin des années 1990. De nombreux travaux rapportent des effets néfastes d'horaires prolongés de travail, à la fois pour les patients ${ }^{[1]}$ (par exemple un accroissement des erreurs médicales ${ }^{[2]}$ ) et pour les médecins résidents (augmentation des blessures par aiguilles ${ }^{[3]}$ et des accidents de la route ${ }^{[4]}$ ).

En 2009, une action en justice a été entreprise au Québec par le président d'une association de médecins résidents, sur le fait que les gardes de 24 heures contreviendraient à la Charte canadienne des droits et libertés de la personne en matière d'emploi, et qu'elles nuisaient à la sécurité des patients. Les tribunaux ont confirmé la légitimité de cette interprétation. En conséquence, et suite à des négociations entreprises entre la Fédération des médecins résidents du Québec (FMRQ) et le gouvernement de la province de Québec, les gardes de 24 heures ont été abolies en 2011 et des modifications majeures dans les horaires de garde ont été mises en place, incluant notamment une interdiction pour les médecins résidents de travailler plus de 16 heures consécutives en milieu hospitalier.

Afin de pouvoir répondre à ces exigences, le programme de résidence en pédiatrie de l'Université de Montréal au centre hospitalier universitaire SainteJustine, à Montréal, a élaboré un nouveau mode de fonctionnement pour les gardes de pédiatrie générale, de néonatalogie et de soins intensifs pédiatriques. Avant le mois d'avril 2012, les résidents de ce programme effectuaient entre quatre et six gardes de 24 heures (de 8 heures à 8 heures le lendemain), par période de quatre semaines, incluant généralement deux gardes de fin de semaine. Les résidents avaient droit à une journée de repos le lendemain de leur garde. Depuis la modification des horaires de gardes, les résidents effectuent plutôt des gardes de 12 heures, soit de $20 \mathrm{~h}$ le soir à $8 \mathrm{~h}$ le matin (avec une période de repos le jour de la garde et une période de repos le lendemain), généralement de manière consécutive. Ils doivent également faire des « périodes-tampons » en soirée (de 17 heures à 20 heures), après leur journée de travail régulière. Par ailleurs, l'organisation des stages cliniques de la résidence en pédiatrie n'a pas été modifiée.

\section{Objectif}

L'objectif de la présente étude est d'évaluer les conséquences de cette modification des horaires de gardes des résidents sur : 1) la perception de la part des résidents de leur qualité de vie (vie sociale, vie familiale, niveau d'anxiété et satisfaction au travail) ; 2) leur perception de la qualité, de la continuité et de la sécurité des soins dispensés aux patients ; 3 ) leur perception de la qualité du programme de résidence.

\section{Méthodes}

\section{Collectifs de participants et administration du questionnaire}

Tous les médecins résidents en pédiatrie ont été rencontrés afin de leur présenter le projet de recherche, d'obtenir leur consentement et de les inviter à répondre à un sondage accessible sur le web. Tous les participants potentiels ont reçu trois appels espacés 
Tableau I. Caractéristiques démographiques des résidents ayant répondu au Q1 et au Q2.

\begin{tabular}{|l|c|c|c|}
\hline & Q1 & Q2 & $p$ \\
\hline Junior, \% & 64,7 & 43,8 & 0,1374 \\
\hline Enfants ou grossesse, \% & 26,5 & 21,9 & 0,7768 \\
\hline En couple, \% & 82,4 & 84,4 & 1,0000 \\
\hline Pratique en pédiatrie générale envisagée, \% & 67,6 & 62,5 & 0,7970 \\
\hline
\end{tabular}

Q1 = Premier questionnaire (avant la modification des horaires de garde).

Q2 = Second questionnaire (après la limitation des horaires de garde).

$p=p$-value (seuil de signification statistique).

de deux semaines. Ce questionnaire a été envoyé une première fois (Q1) en février et mars 2012, soit avant le changement du modèle de garde dans le programme. Le Q1 comportait 55 questions incluant : a) cinq questions sur les caractéristiques démographiques du sujet ; b) sept questions sur les modalités de travail en place ; c) 18 questions sur leur perception de la qualité de vie (vie sociale, santé, sommeil, sécurité et bien-être) ; d) 12 questions sur leur perception de la sécurité, de la continuité et de la qualité dans les soins aux patients ; e) 12 questions sur l'appréciation du programme de formation. Un espace était également réservé pour les commentaires libres.

Le même questionnaire a été envoyé aux résidents une seconde fois (Q2), selon la même procédure, en octobre et novembre 2012, soit sept à huit mois après la transition vers le nouveau modèle de garde. Certains résidents avaient entre temps été diplômés et une nouvelle cohorte de résidents est entrée dans le programme de pédiatrie durant cette période.

Compte tenu du caractère sensible de certaines questions, tous les questionnaires ont inclus les coordonnées du programme d'aide aux médecins du Québec (PAMQ), afin que les résidents qui prendraient conscience de leurs difficultés en répondant au questionnaire puissent obtenir le soutien approprié grâce à un service confidentiel et extérieur au programme de résidence.

Toutes les données ont été compilées électroniquement puis rendues anonymes afin d'éviter l'identification des résidents lors de l'analyse des résultats. L'objectif était d'assurer la confidentialité des résultats afin que les résidents puissent répondre aux questionnaires en toute liberté et sans crainte de conséquences.

\section{Analyse statistique}

Les données ont été analysées à l'aide de statistiques descriptives et du test de Fisher exact. Le seuil de signification statistique $\alpha$ est établi à 0,05 .

\section{Résultats}

Parmi les 40 résidents, 34 résidents ont répondu au Q1 (taux de réponse de $85 \%$ ) alors que 32 sur 40 ont complété le Q2 (taux de réponse de $80 \%$ ).

\section{Données démographiques}

Le pourcentage de résidents juniors (de $1^{\text {ère }}$ ou de $2^{\mathrm{e}}$ année sur un programme d'une durée de quatre ou cinq ans) était de 64,7 \% parmi les répondants au Q1. Ce pourcentage s'établit à $43,8 \%$ pour le Q2. La majorité prévoit une carrière en pédiatrie générale. Environ $80 \%$ des résidents vivent en couple et le quart ont des enfants ou sont enceintes. Il n'y a pas de différence statistiquement significative entre les caractéristiques démographiques des répondants aux deux questionnaires (tableau I). Le sexe des répondants n'était pas inclus dans les questions en raison du très petit nombre d'hommes au sein de ce programme de formation et d'un désir que les répondants ne puissent être aisément identifiés par les 
Tableau II. Modalités de travail.

\begin{tabular}{|c|c|c|c|c|c|}
\hline \multicolumn{6}{|c|}{ Nombres d'heures (h)/semaine en } \\
\hline \multirow{5}{*}{ Q1 } & $>80 \mathrm{~h}$ & $4(11,8)$ & \multirow{5}{*}{ Q2 } & $>80 \mathrm{~h}$ & 0 \\
\hline & $71-80 \mathrm{~h}$ & $13(38,2)$ & & $71-80 \mathrm{~h}$ & $5(15,6)$ \\
\hline & $61-70 \mathrm{~h}$ & $15(44,1)$ & & $61-70 \mathrm{~h}$ & $18(56,3)$ \\
\hline & $51-60 \mathrm{~h}$ & $2(5,9)$ & & $51-60$ & $9(28,1)$ \\
\hline & $\leq 50 \mathrm{~h}$ & 0 & & $\leq 50 \mathrm{~h}$ & 0 \\
\hline \multicolumn{6}{|c|}{ Nombres d'heures (h)/semaine d'étude à l'extérieur de l'hôpital $n(\%)$} \\
\hline \multirow{5}{*}{ Q1 } & $<1 \mathrm{~h}$ & $5(14,7)$ & \multirow{5}{*}{ Q2 } & $<1 \mathrm{~h}$ & $2(6,3)$ \\
\hline & $1-5 \mathrm{~h}$ & $17(50)$ & & $1-5 \mathrm{~h}$ & $12(37,5)$ \\
\hline & $6-10 \mathrm{~h}$ & $7(20,6)$ & & $6-10 \mathrm{~h}$ & $10(31,2)$ \\
\hline & $11-20 \mathrm{~h}$ & $3(8,8)$ & & $11-20 \mathrm{~h}$ & $3(9,4)$ \\
\hline & $>20 \mathrm{~h}$ & $2(5,9)$ & & $>20 \mathrm{~h}$ & $5(15,6)$ \\
\hline
\end{tabular}

Q1 = Premier questionnaire (avant la modification des horaires de garde).

$\mathrm{Q} 2=$ Second questionnaire (après la limitation des horaires de garde).

chercheurs lors de l' analyse des résultats. À titre indicatif, lors de l'administration du Q1, le programme de pédiatrie comportait seulement quatre hommes sur un total de 40 résidents.

\section{Modalités de travail}

Avant le changement des horaires de garde, le nombre médian de gardes était de cinq gardes de 24 heures par période de quatre semaines. Après le passage au système de 12 heures, le nombre médian de gardes par période de quatre semaines est également de cinq gardes, mais de 12 heures chacune, avec trois gardes supplémentaires en soirée $(17 \mathrm{~h}$ à 20 h). Le nombre d'heures de travail à l'hôpital est de 61 à 70 heures par semaine pour la majorité des répondants, avant comme après le changement des horaires de garde $(44,1 \%$ versus $53,1 \%$ respectivement, $p=0,6225)$. On constate une tendance à l'augmentation du temps disponible pour l'étude en dehors du temps clinique depuis la réforme des horaires (tableau II).

\section{Perception de la qualité de vie et du bien-être}

Dans le Q1, 85,3 \% (29/34) des résidents ont répondu que leur qualité de vie s'était détériorée depuis le début de leur résidence. Après le changement du système de garde, $50 \%$ affirment que leur qualité de vie s'était améliorée et un tiers ne note aucun changement dans leur qualité de vie après le changement du système de garde.

Dans le Q2, $62 \%$ des résidents s'attendaient à une amélioration de leur qualité de vie grâce au passage aux gardes de 12 heures et $84 \%$ confirment cette donnée dans le Q2.

Pour $87,5 \%$ des résidents sondés initialement, leur vie sociale s'était détériorée depuis le début de la résidence. Suite à l'abolition des gardes de 24 heures, le pourcentage qui déclare une amélioration de leur vie sociale aux cours des six mois précédents s'élève à $71,9 \%$.

Les résidents ont été questionnés sur leur perception de la qualité de leur vie familiale. La moitié des résidents $(50 \%)$ mentionnent une diminution de la qualité perçue de vie familiale depuis qu'ils ont débuté la résidence. Six mois après le changement, le tiers des résidents souligne une amélioration récente de leur vie familiale. Près de $45 \%$ des résidents ayant des enfants au moment du Q1 affirmaient que le changement du système de gardes serait néfaste pour la qualité de vie des résidents ; $14 \%$ d'entre eux sont du même avis lors du Q2.

Dans le Q1, 35,3\% des répondants ont déclaré que leur état de santé s'était détérioré depuis le début 


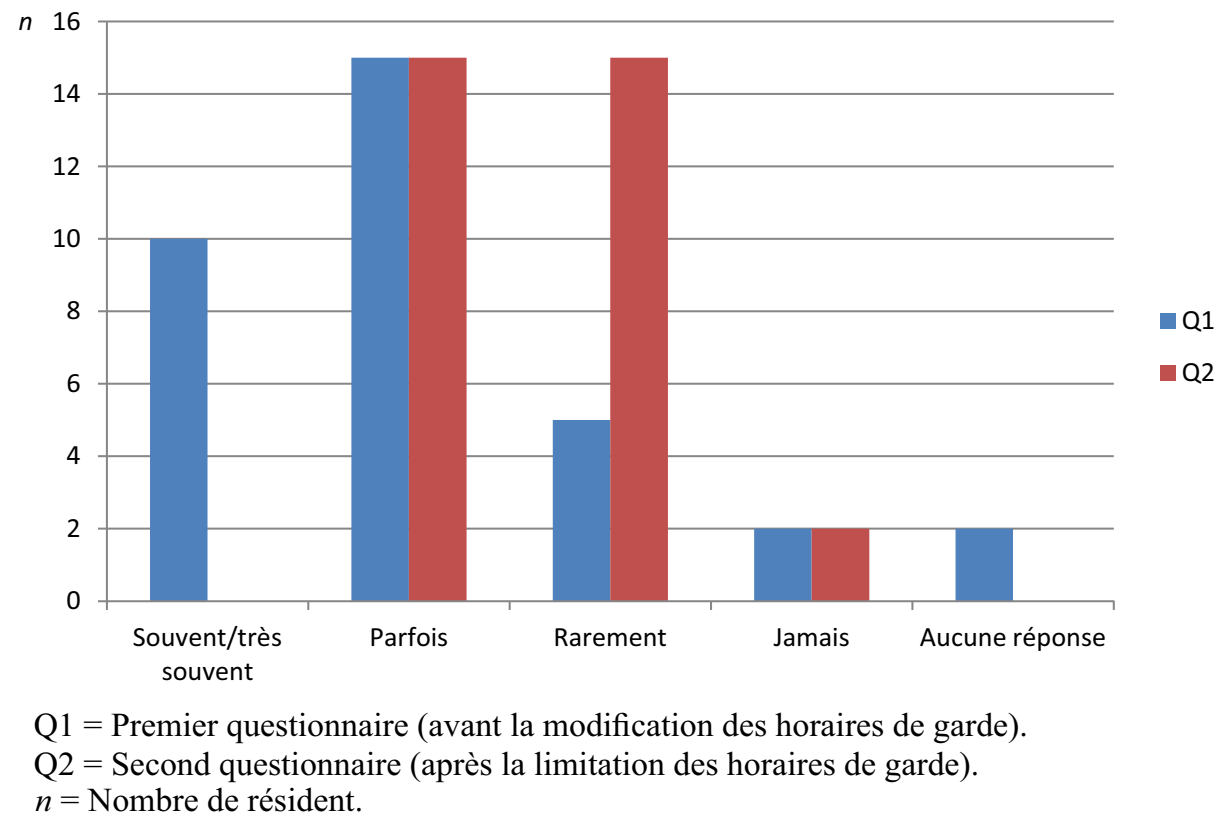

Fig. 1. Réponses à la question : « J'ai eu l'impression que ma fatigue compromettait la sécurité de mes patients ».

de leur résidence (contre 58,8\% dont l'état de santé était resté inchangé). Lors du Q2, 28,1\% des répondants soulignent que leur état de santé s'est amélioré depuis les six derniers mois. Pour la majorité d'entre eux, leur état de santé n'a pas changé $(65,6 \%)$.

Un nombre important de résidents rapportent une humeur triste persistante : près d'un quart dans le Q1 $(20,6 \%)$ et $6,3 \%$ dans le Q2 $(p=0,1526)$. Pour ce qui est de l'anxiété persistante, ces pourcentages sont respectivement de $50 \%$ et $15,7 \%(p=0,0041)$.

Dans le Q1, $75 \%$ des répondants déclaraient être très souvent ou souvent fatigués tandis que dans le Q2 ce nombre baisse à 62,5\% ( $p=0,0213)$.

Avant le changement des horaires de gardes, $29,4 \%$ des résidents décrivaient des troubles du sommeil alors qu'ils sont 15,6\% dans le Q2, la différence étant statistiquement significative $(p=0,0374)$.

Perception de la sécurité, de la continuité et de la qualité dans les soins

Avant la modification du système de garde, $88 \%$ des résidents pensaient que l'abolition des gardes de
24 heures aurait pour effet d'améliorer la sécurité de leurs patients. Ce pourcentage augmente à $96 \%$ quand on leur pose la même question après le changement. Les résidents ont été questionnés quant à la fatigue perçue pendant les gardes et au risque qu'elle compromette la sécurité des patients. Les réponses à cette question sont présentées sur la figure 1 .

\section{Appréciation du programme de formation}

La satisfaction des résidents concernant leur présence dans les stages cliniques a été améliorée de façon statistiquement significative après la modification des horaires de garde : degré de satisfaction excellent ou bon chez $32,3 \%(\mathrm{Q} 1)$ versus $62,5 \%(\mathrm{Q} 2)$ des résidents $(p=0,0256)$.

\section{Discussion}

Cette étude montre que le nombre moyen d'heures travaillées par semaine en milieu hospitalier est le même avant et après le changement pour la majorité 


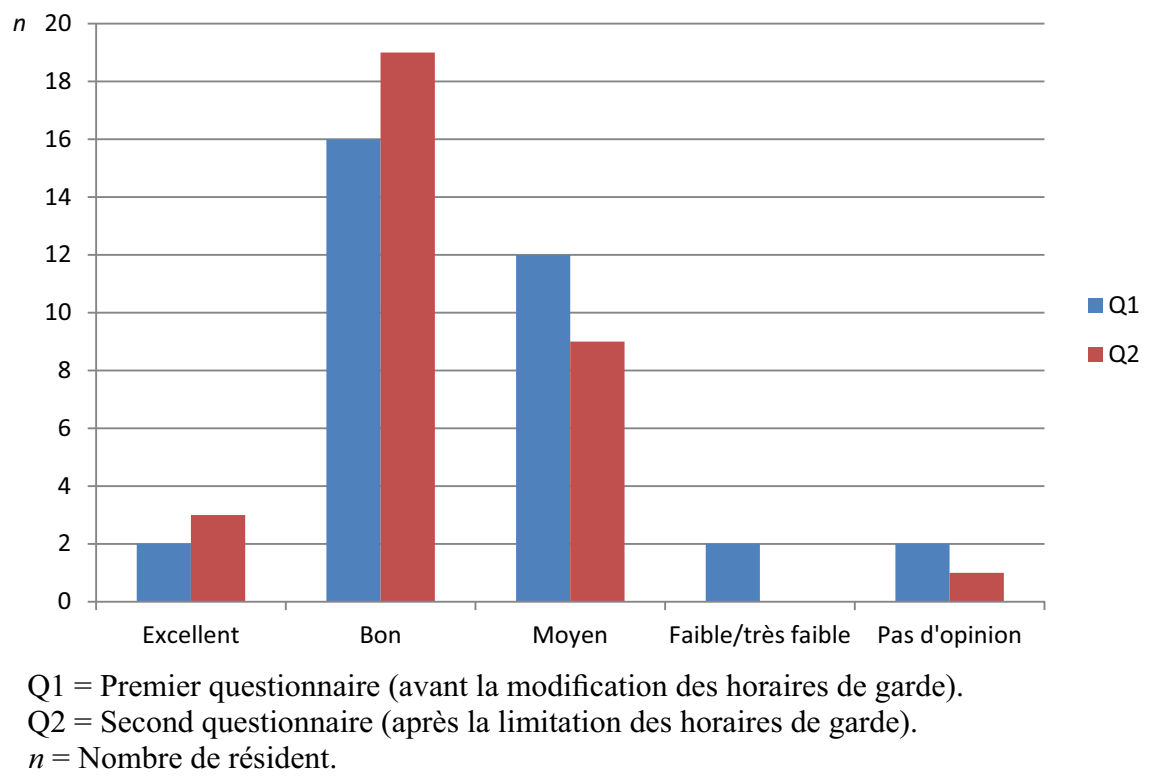

Fig. 2. Degré de satisfaction des résidents envers leur programme de résidence.

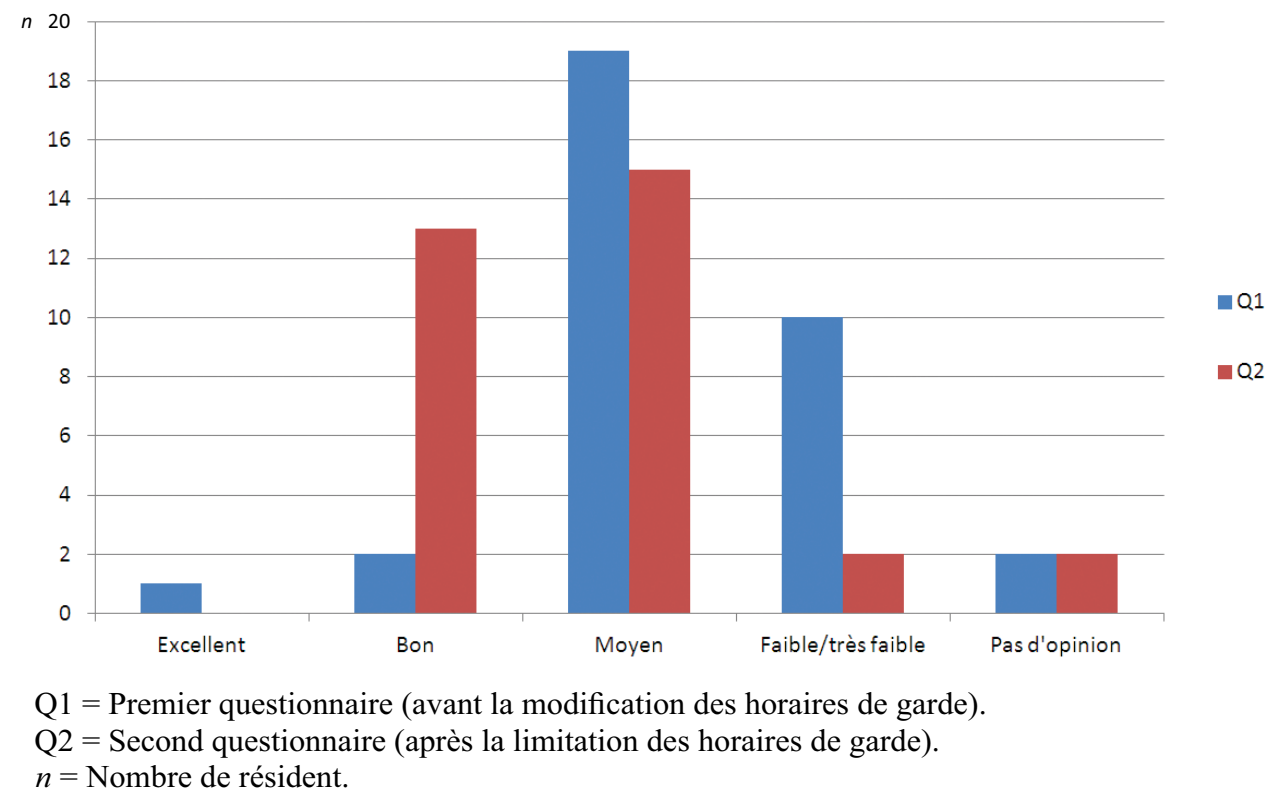

Fig. 3. Degré de satisfaction rapporté par les résidents envers les gardes de leur programme de résidence.

des répondants. Une majorité (62\%) des résidents prévoyait une amélioration de leur qualité de vie avec l'abolition des gardes de 24 heures (Q1) alors que $84 \%$ pensaient que le nouveau système de garde avait amélioré leur qualité de vie dans le Q2. Pour $96 \%$ des répondants, la sécurité des patients s'est améliorée après le changement du système de garde.

L'amélioration de la qualité de vie et du bien-être perçus des résidents suite au changement du système de garde est soulignée dans notre étude et rejoint les données d'études précédentes ${ }^{[5,6]}$. 
Le pourcentage de résidents qui se plaignent de fatigue souvent ou très souvent est de $75 \%$ dans notre étude. Ce pourcentage chute de manière significative après le changement du modèle de garde mais il demeure élevé $(62,5 \%)$. Les mesures visant à réduire les heures de garde et/ou la fréquence des gardes se montrent efficaces pour prévenir la fatigue ${ }^{[7]}$ et les baisses de l'attention ${ }^{[8]}$. Les mesures de réduction des heures de garde consécutives semblent donc pouvoir contribuer à la sécurité des soignants comme des soignés.

Les résidents n'ont pas été questionnés spécifiquement sur les heures de sommeil avant et après le changement de système de garde. Cependant les troubles du sommeil semblent être fréquents chez les résidents du programme $(29,4 \%)$. Ce pourcentage est réduit de moitié dans le Q2. Les effets délétères de la privation chronique de sommeil ${ }^{[9]}$ sont bien connus dans la littérature scientifique : obésité ${ }^{[10]}$, altération des performances cognitives, humeur dépressive $^{[11]}$ et même mortalité accrue ${ }^{[12]}$. Les résidents de différentes spécialités ont identifié le manque de sommeil et la fatigue comme ayant un impact majeur sur leur vie personnelle pendant la résidence et comme ayant des conséquences sur leurs capacités d'apprentissage, leur performance au travail et leur professionnalisme ${ }^{[12]}$.

Les enjeux de santé mentale (humeur dépressive et anxiété en particulier) semblent majeurs au sein de ce programme de formation. Dans d'autres programmes de résidences en pédiatrie, Landrigan et al. ${ }^{[13]}$ ont décrit des taux importants de dépression et d'épuisement professionnel. Dans cette étude prospective, ils ont démontré une amélioration des taux d'épuisement professionnel mais pas de changement des taux de dépression suite aux restrictions des heures de gardes aux États-Unis. Cette amélioration a aussi été notée en spécialités chirurgicales ${ }^{[6]}$.

Compte tenu de ces multiples préoccupations liées aux conséquences des horaires prolongés de travail, les systèmes de gardes des résidents se réinventent, de gré ou de force. Plusieurs médecins superviseurs sont toutefois préoccupés par les conséquences de ces réductions des heures de gardes, craignant une déresponsabilisation des résidents dans les soins aux patients, une réduction des opportunités d'enseignement et d'apprentissage (nuisant à la formation des résidents) et une réduction dans la continuité des soins ${ }^{[14]}$. Notre sondage semble montrer au contraire que les résidents perçoivent une meilleure continuité de leur présence en stages cliniques. Les études disponibles ${ }^{[1]}$ jusqu'à maintenant ne semblent pas montrer d'effet néfaste sur la dimension pédagogique des stages mais aucune n'a étudié spécifiquement l'impact de gardes de 12 heures. De façon générale, le lien entre restriction des heures de travail et rendement académique des résidents demeure un sujet de controverse.

Les médecins résidents ont le double rôle d'apprenants et de soignants. Dans les centres hospitaliers universitaires, les résidents sont impliqués directement dans les soins courants des patients. L'objectif d'un système de garde idéal est d'offrir un équilibre entre les apprentissages et les services rendus aux patients par les résidents. Un système de gardes de 12 heures semble améliorer la perception de la qualité des soins offerts et accroît également la satisfaction à l'égard du programme de formation. D'autres études devraient tenter de mesurer objectivement les effets du changement des horaires de gardes, en particulier avec un système de gardes de 12 heures, sur la qualité des soins qui sont dispensés aux patients. Pour l'instant, la littérature ne fournit aucune preuve solide que la diminution du nombre d'heures de travail consécutives est nécessaire pour assurer la sécurité des patients. La perception des résidents de notre programme semble cependant favoriser un système de gardes de 12 heures.

\section{Points forts de l'étude}

Le questionnaire administré a permis de démontrer des différences statistiquement significatives concernant les réponses formulées par les participants à plusieurs questions, respectivement aux deux moments de l'étude et ce, malgré un nombre limité de participants. Ces résultats contribuent ainsi à documenter l'impact d'un changement de modèle de gardes sur la perception qu'ont les résidents des soins donnés, de leur qualité de vie et de la qualité de leur formation médicale. Contrairement à de nombreux articles sur 
le sujet, notre étude a comparé les horaires de garde de 24 heures avec ceux de 12 heures, alors que la plupart des auteurs nord-américains étudient les horaires de 16 heures consécutives.

Limites de l'étude

Notre étude comporte certaines faiblesses méthodologiques, liées notamment aux limites du questionnaire qui a été administré. En raison de contraintes importantes de temps liées à l'instauration des nouvelles modalités de garde, il n'a pas été possible de réaliser une étude pilote préalable, qui aurait notamment permis de vérifier les qualités psychométriques du questionnaire. Cependant, les thèmes explorés par le questionnaire ont été identifiés à partir d'une revue de la littérature disponible sur la modification des horaires de garde chez les médecins résidents.

Les caractéristiques des non-répondants de cette étude ne sont par ailleurs pas connues. Il n'est donc pas possible de comparer les groupes des répondants et des non-répondants et de discuter les conséquences de ce biais sur les résultats.

Le nombre de sujets étudiés étant petit, la puissance des procédures statistiques est limitée. Il serait pertinent d'envisager des études avec un nombre plus important de sujets afin de définir les caractéristiques des résidents (parentalité, statut marital, sexe, niveau de formation et autres caractéristiques individuelles) qui pourraient être corrélées à leurs perceptions des conséquences des modèles de garde sur leur qualité de vie.

Le devis de l'étude ne permet pas de conclure à des liens de causalité entre la modification des horaires de gardes et les tendances observées.

Enfin entre les deux périodes de l'étude, certains résidents ont été diplômés et ont donc quitté le programme, alors que d'autres ont commencé leur résidence. La cohorte de nouveaux résidents de première année était importante, ce qui a pu modifier la charge de travail pour chaque résident, notamment sur les gardes. Il s'agit là d'un biais potentiel. Les caractéristiques démographiques des deux groupes d'étude sont par contre semblables.

\section{Conclusion}

Dans un programme de résidence en pédiatrie québécois, la suppression des gardes de 24 heures a eu des impacts principalement positifs sur les perceptions des résidents relativement à leur qualité de vie et à l'impression de sécurité et de qualité des soins dispensés aux patients. La limitation du nombre d'heures consécutives de travail à 12 heures n'est pas associée à une réduction significative du nombre d'heures total de travail par semaine pour les résidents. Les résidents décrivent une amélioration de leur qualité de vie perçue suite à la modification des horaires de gardes.

\section{Contributions}

Marie-Hélène Lizotte a contribué à la conception du protocole de recherche, au recueil des données, à l'interprétation des résultats, à l'analyse statistique et à l'écriture du manuscrit. Marie-Christine Lamy a participé à la conception du protocole de recherche et au recueil des données. Benoit Carrière a participé à la conception du protocole de recherche, au recueil des données, à l'interprétation des résultats, à l'analyse statistique et à l'écriture du manuscrit

\section{Approbation éthique}

Le projet a été approuvé par le comité d'éthique de la recherche du centre hospitalier universitaire SainteJustine.

\section{Déclaration d'intérêts}

M-HL déclare n'avoir aucun conflit d'intérêts, M-C.L déclare n'avoir aucun conflit d'intérêts, $\mathrm{BC}$ déclare n'avoir aucun conflit d'intérêts. 


\section{Références}

1. Levine AC, Adusumilli J, Landrigan CP. Effects of reducing or eliminating resident work shifts over 16 hours: a systematic review. Sleep 2010;33:104353.

2. Landrigan CP, Rothschild JM, Cronin JW, Kaushal R, Burdick E, Katz JT, et al. Effect of reducing interns' work hours on serious medical errors in intensive care units. N Engl J Med 2004;351:1838-48.

3. Ayas NT, Barger LK, Cade BE, Hashimoto DM, Rosner B, Cronin JW, et al. Extended work duration and the risk of self-reported percutaneous injuries in interns. JAMA 2006;296:1055-62.

4. Barger LK, Cade BE, Ayas NT, Cronin JW, Rosner B, Speizer FE et al. Extended work shifts and the risk of motor vehicle crashes among interns. N Engl J Med 2005;352:125-34.

5. Myers JS, Bellini LM, Morris JB, Graham D, Katz J, Potts JR, et al. Internal medicine and general surgery residents' attitudes about the ACGME duty hours regulations: a multicenter study. Acad Med 2006;81: 1052-8

6. Lindeman BM, Sacks BC, Hirose K, Lipsett PA. Multifaceted longitudinal study of surgical resident education, quality of life, and patient care before and after July 2011. J Surg Educ 2013; 70:769-76.

7. Sawyer RG, Tribble CG, Newberg DS, Pruett TL, Minasi JS. Intern call schedules and their relationship to sleep, operating room participation, stress, and satisfaction. Surgery 1999;126:337-42 .
8. Lockley SW, Cronin JW, Evans EE, Cade BE, Lee CJ, Landrigan CP et al. Effect of reducing interns' weekly work hours on sleep and attentional failures. N Engl J Med 2004;351:1829-37.

9. Olson EJ, Drage LA, Auger RR. Sleep deprivation, physician performance, and patient safety. Chest 2009;136:1389-96.

10. Cappuccio FP, Taggart FM, Kandala NB, Currie A, Peile E, Stranges S, et al. Meta-analysis of short sleep duration and obesity in children and adults. Sleep 2008;31:619-26.

11. Rosen IM, Gimotty PA, Shea JA, Bellini LM. Evolution of sleep quantity, sleep deprivation, mood disturbances, empathy, and burnout among interns. Acad Med 2006;81:82-5.

12. Papp KK, Stoller EP, Sage P, Aikens JE, Owens J, Avidan A, et al. The effects of sleep loss and fatigue on resident-physicians: a multi-institutional, mixedmethod study. Acad Med 2004;79:394-406.

13. Landrigan CP, Fahrenkopf AM, Lewin D, Kaushal R, Burdick E, Katz JT, et al. Effects of the accreditation council for graduate medical education duty hour limits on sleep, work hours, and safety. Pediatrics 2008; 122:250-8.

14. Drolet BC, Whittle SB, Khokhar MT, Fischer SA, Pallant A. Approval and perceived impact of duty hour regulations: survey of pediatric program directors. Pediatrics 2013;132:819-24.

Correspondance et offprints : Marie-Hélène Lizotte, Université de Montréal, CHU Sainte-Justine, 3175 Chemin de la CôteSainte-Catherine, Montréal, QC H3T 1C5. 514-345-4931 poste 4788.

Mailto : marie-helene.lizotte-masson@umontreal.ca 